ANNALES

POLONICI MATHEMATICI

$98.3(2010)$

\title{
3-submersions from QR-hypersurfaces of quaternionic Kähler manifolds
}

\author{
by Gabriel Eduard Vîlcu (Ploieşti and Bucureşti)
}

\begin{abstract}
We study 3-submersions from a QR-hypersurface of a quaternionic Kähler manifold onto an almost quaternionic hermitian manifold. We also prove the non-existence of quaternionic submersions between quaternionic Kähler manifolds which are not locally hyper-Kähler.
\end{abstract}

1. Introduction. In WTS B. Watson introduced the notion of 3submersion, as a Riemannian submersion from an almost contact metric manifold onto an almost quaternionic manifold, which commutes with the structure tensors of type $(1,1)$. In [IMV1] and [IMV2, this concept has been extended in quaternionic setting. In this paper we study 3 -submersions from QR-hypersurfaces of quaternionic Kähler manifolds, we give an example and obtain some obstructions to the existence of quaternionic submersions.

The study of QR-submanifolds of a quaternionic Kähler manifold was initiated by A. Bejancu BJC]. Among all submanifolds of a quaternionic Kähler manifold, QR-submanifolds have been intensively studied by several authors [AG, BEJ, BF, GS, KP, KPK, MNG, SHN1, SHN2]. In Section 2 we recall the definitions and basic properties of quaternionic manifolds and QR-submanifolds of a quaternionic Kähler manifold.

On the other hand, R. Güneş, B. Şahin and S. Keleş [GS] have shown that a QR-submanifold admits an almost contact 3-structure under some conditions. In Section 3 we see that on an orientable hypersurface of a quaternionic Kähler manifold there exists a natural almost contact metric 3-structure. This result will allow us to define the concept of QR 3-submersion. In Section 4 we obtain some properties for this kind of submersions and give an example. In the last section we prove the non-existence of quaternionic submersions between quaternionic Kähler non-locally hyper-Kähler manifolds.

2010 Mathematics Subject Classification: Primary 53C15; Secondary 53C26.

Key words and phrases: Riemannian submersion, quaternionic Kähler manifold, QRsubmanifold. 
2. Preliminaries. Let $M$ be a differentiable manifold of dimension $n$ and assume that there is a rank 3-subbundle $\sigma$ of $\operatorname{End}(T M)$ which is locally spanned by an almost hypercomplex structure, i.e. a triple $\left\{J_{1}, J_{2}, J_{3}\right\}$ of almost complex structures satisfying the quaternionic identities:

$$
\left\{\begin{array}{l}
J_{\alpha}^{2}=-\mathrm{Id}, \quad \forall \alpha \in\{1,2,3\}, \\
J_{1} J_{2}=-J_{2} J_{1}=J_{3} .
\end{array}\right.
$$

Then the bundle $\sigma$ is called an almost quaternionic structure on $M$ and $\left\{J_{1}, J_{2}, J_{3}\right\}$ is called a canonical local basis of $\sigma$. Moreover, $(M, g)$ is then said to be an almost quaternionic manifold. It is easy to see that any almost quaternionic manifold is of dimension $n=4 \mathrm{~m}$.

A Riemannian metric $g$ is adapted to the quaternionic structure $\sigma$ if

$$
g\left(J_{\alpha} X, J_{\alpha} Y\right)=g(X, Y), \quad \forall \alpha \in\{1,2,3\},
$$

for all vector fields $X, Y$ on $M$ and any local basis $\left\{J_{1}, J_{2}, J_{3}\right\}$ of $\sigma$. Moreover, $(M, \sigma, g)$ is then said to be an almost quaternionic hermitian manifold.

If the bundle $\sigma$ is parallel with respect to the Levi-Civita connection $\nabla$ of $g$, then $(M, \sigma, g)$ is said to be a quaternionic Kähler manifold. Equivalently, there exists locally defined 1-forms $\omega_{1}, \omega_{2}, \omega_{3}$ such that

$$
\left\{\begin{array}{l}
\nabla_{X} J_{1}=\omega_{3}(X) J_{2}-\omega_{2}(X) J_{3}, \\
\nabla_{X} J_{2}=-\omega_{3}(X) J_{1}+\omega_{1}(X) J_{3}, \\
\nabla_{X} J_{3}=\omega_{2}(X) J_{1}-\omega_{1}(X) J_{2},
\end{array}\right.
$$

for any vector field $X$ on $M$. In particular, if $\omega_{1}=\omega_{2}=\omega_{3}=0$, then $(M, \sigma, g)$ is said to be a locally hyper-Kähler manifold.

We remark that any quaternionic Kähler manifold $M$ is an Einstein space, provided that $\operatorname{dim} M>4$. Moreover, $M$ is irreducible (if Ric $\neq 0$ ) or locally hyper-Kähler manifold (if Ric $=0$ ) (see [AL, BES, ISH, SLM]).

Let $(M, \sigma, g)$ be an almost quaternionic hermitian manifold. If $X \in T_{p} M$, $p \in M$, then the 4-plane $Q(X)$ spanned by $\left\{X, J_{1} X, J_{2} X, J_{3} X\right\}$ is called a quaternionic 4-plane. A 2-plane in $T_{p} M$ spanned by $\{X, Y\}$ is called halfquaternionic if $Q(X)=Q(Y)$.

The sectional curvature for a half-quaternionic 2-plane is called quaternionic sectional curvature. A quaternionic Kähler manifold is a quaternionic space form if its quaternionic sectional curvatures are equal to a constant, say $c$. It is well-known that a quaternionic Kähler manifold $(M, \sigma, g)$ is a quaternionic space form (denoted $M(c)$ ) if and only if its curvature tensor is

$$
\begin{aligned}
R(X, Y) Z= & \frac{c}{4}\left\{g(Z, Y) X-g(X, Z) Y+\sum_{\alpha=1}^{3}\left[g\left(Z, J_{\alpha} Y\right) J_{\alpha} X\right.\right. \\
& \left.\left.-g\left(Z, J_{\alpha} X\right) J_{\alpha} Y+2 g\left(X, J_{\alpha} Y\right) J_{\alpha} Z\right]\right\}
\end{aligned}
$$

for all vector fields $X, Y, Z$ on $M$ and any local basis $\left\{J_{1}, J_{2}, J_{3}\right\}$ of $\sigma$. 
Let $(\bar{M}, \sigma, \bar{g})$ be a quaternionic Kähler manifold and let $M$ be a real submanifold of $\bar{M}$. Then $M$ is said to be a $Q R$-submanifold if there exists a vector subbundle $D$ of the normal bundle $T M^{\perp}$ such that:

(i) $J_{\alpha}\left(D_{p}\right)=D_{p}$ for all $p \in M$ and $\alpha \in\{1,2,3\}$;

(ii) $J_{\alpha}\left(D_{p}^{\perp}\right) \subset T_{p} M$ for all $p \in M$ and $\alpha \in\{1,2,3\}$, where $D^{\perp}$ is the complementary orthogonal bundle to $D$ in $T M^{\perp}$ (see [BJC]).

3. QR-hypersurfaces and almost contact metric 3-structures. Let $M$ be an orientable hypersurface of a quaternionic Kähler manifold $(\bar{M}, \sigma, \bar{g})$ and $\xi$ a unit normal field on $M$. If we take $D=0$, then $D^{\perp}=T M^{\perp}$ and we conclude that $M$ is a QR-submanifold of $\bar{M}$.

Let $\left\{J_{\alpha}\right\}_{\alpha \in\{1,2,3\}}$ and $\left\{J_{\alpha}^{\prime}\right\}_{\alpha \in\{1,2,3\}}$ be two local bases defined on coordinate neighborhoods $\bar{U}$ and $\bar{U}^{\prime}$ with $\bar{U} \cap \bar{U}^{\prime} \neq \emptyset$. Then, on $\bar{U}$,

$$
\xi_{\alpha}=-J_{\alpha} \xi, \quad \forall \alpha \in\{1,2,3\},
$$

defines tangent vector fields to $M$ and similarly, on $\bar{U}^{\prime}$,

$$
\xi_{\alpha}^{\prime}=-J_{\alpha}^{\prime} \xi, \quad \forall \alpha \in\{1,2,3\},
$$

defines tangent vector fields to $M$.

Moreover, on $\bar{U} \cap \bar{U}^{\prime}$ we have

$$
\xi_{\alpha}^{\prime}=\sum_{\beta=1}^{3} c_{\alpha \beta} \xi_{\beta}, \quad \forall \alpha \in\{1,2,3\},
$$

where $C=\left(c_{\alpha \beta}\right)_{\alpha, \beta \in\{1,2,3\}} \in S O(3)$. Thus, we obtain a distribution $\mathcal{V}$ on $M$, which is locally generated by $\left\{\xi_{\alpha}\right\}_{\alpha \in\{1,2,3\}}$. Let $\mathcal{H}$ be the orthogonal complementary distribution to $\mathcal{V}$ with respect to the Riemannian metric $g$ induced by $\bar{g}$ on $M$. We remark that for each $p \in M, \mathcal{H}_{p}$ is $J_{\alpha}$-invariant for all $\alpha \in\{1,2,3\}$.

We recall that the distribution $\mathcal{V}$ is integrable if and only if $M$ is a mixed geodesic QR-hypersurface of $\bar{M}$, i.e.

$$
B(U, X)=0, \quad \forall U \in \Gamma(\mathcal{V}), \forall X \in \Gamma(\mathcal{H}),
$$

where $B$ is the second fundamental form of $M$ in $\bar{M}$ (see [BJC]).

Definition 3.1 ([BLR $]$ ). Let $M$ be a differentiable manifold equipped with a triple $(\phi, \xi, \eta)$, where $\phi$ is a field of endomorphisms of tangent spaces, $\xi$ is a vector field and $\eta$ is a 1 -form on $M$. If

$$
\phi^{2}=-I+\eta \otimes \xi, \quad \eta(\xi)=1,
$$

then we say that $(\phi, \xi, \eta)$ is an almost contact structure on $M$.

Definition 3.2 ([KU] $)$. Let $M$ be a differentiable manifold which admits three almost contact structures $\left(\phi_{\alpha}, \xi_{\alpha}, \eta_{\alpha}\right), \alpha \in\{1,2,3\}$, satisfying the 
following conditions:

$$
\begin{aligned}
\eta_{\alpha}\left(\xi_{\beta}\right) & =0, \quad \forall \alpha \neq \beta, \\
\phi_{\alpha}\left(\xi_{\beta}\right) & =-\phi_{\beta}\left(\xi_{\alpha}\right)=\xi_{\gamma}, \\
\eta_{\alpha} \circ \phi_{\beta} & =-\eta_{\beta} \circ \phi_{\alpha}=\eta_{\gamma}, \\
\phi_{\alpha} \phi_{\beta}-\eta_{\beta} \otimes \xi_{\alpha} & =-\phi_{\beta} \phi_{\alpha}+\eta_{\alpha} \otimes \xi_{\beta}=\phi_{\gamma},
\end{aligned}
$$

where in (3.4)-(3.6),$(\alpha, \beta, \gamma)$ is an even permutation of $(1,2,3)$. Then the manifold $M$ is said to have an almost contact 3 -structure $\left(\phi_{\alpha}, \xi_{\alpha}, \eta_{\alpha}\right)_{\alpha \in\{1,2,3\}}$.

Definition 3.3 ([KUO]). Let $(M, g)$ be a Riemannian manifold endowed with an almost contact 3-structure $\left(\phi_{\alpha}, \xi_{\alpha}, \eta_{\alpha}\right)_{\alpha \in\{1,2,3\}}$ such that

$$
\begin{aligned}
\eta_{\alpha}(X) & =g\left(X, \xi_{\alpha}\right), \quad \forall \alpha \in\{1,2,3\}, \\
g\left(\phi_{\alpha} X, \phi_{\alpha} Y\right) & =g(X, Y)-\eta_{\alpha}(X) \eta_{\alpha}(Y), \quad \forall \alpha \in\{1,2,3\},
\end{aligned}
$$

for all vector fields $X, Y$ on $M$. Then we say that $M$ admits an almost contact metric 3-structure.

Definition 3.4 ([BLR]). We say that an almost contact metric 3-structure $\left(\phi_{\alpha}, \xi_{\alpha}, \eta_{\alpha}\right)_{\alpha \in\{1,2,3\}}$ on a Riemannian manifold $(M, g)$ is a 3 -cosymplectic structure if

$$
\left(\nabla_{X} \phi_{\alpha}\right)(Y)=0, \quad\left(\nabla_{X} \eta_{\alpha}\right)(Y)=0, \quad \forall \alpha \in\{1,2,3\} .
$$

Let $M$ be an orientable hypersurface of a quaternionic Kähler manifold $\bar{M}$. If $S: T M \rightarrow \mathcal{H}$ is the canonical projection, then any local vector field $X$ on $M$ can be expressed as follows:

$$
X=S X+\sum_{\alpha=1}^{3} \eta_{\alpha}(X) \xi_{\alpha}
$$

where

$$
\eta_{\alpha}(X)=g\left(X, \xi_{\alpha}\right), \quad \forall \alpha \in\{1,2,3\} .
$$

From (3.10) we have

$$
J_{\alpha} X=J_{\alpha} S X+\sum_{\beta=1}^{3} \eta_{\beta}(X) J_{\alpha} \xi_{\beta}, \quad \forall \alpha \in\{1,2,3\} .
$$

From $(3.12)$ we obtain the decomposition

$$
J_{\alpha} X=\phi_{\alpha} X+F_{\alpha} X,
$$

where $\phi_{\alpha} X$ is the tangential part of $J_{\alpha} X$ given by

$$
\phi_{\alpha} X=J_{\alpha} S X+\eta_{\beta}(X) \xi_{\gamma}-\eta_{\gamma}(X) \xi_{\beta},
$$

and $F_{\alpha} X$ is the normal part of $J_{\alpha} X$ given by

$$
F_{\alpha} X=\eta_{\alpha}(X) \xi
$$

for all $\alpha \in\{1,2,3\}$, where $(\alpha, \beta, \gamma)$ is an even permutation of $(1,2,3)$. 
By straightforward computations, we can easily see that $\left(\phi_{\alpha}, \xi_{\alpha}, \eta_{\alpha}\right)_{\alpha}$ defined by (3.11), (3.14) and (3.15) is an almost contact metric 3-structure on $M$ and so we have the next result (see also [GS]).

Proposition 3.5. Any QR-hypersurface of a quaternionic Kähler manifold admits a natural almost contact metric 3-structure.

\section{3-submersions of QR-hypersurfaces}

Definition 4.1. Let $M$ be a mixed geodesic QR-hypersurface of a quaternionic Kähler manifold $\bar{M}$ endowed with the natural almost contact metric 3 -structure $\left(\phi_{\alpha}, \xi_{\alpha}, \eta_{\alpha}\right)_{\alpha \in\{1,2,3\}}$ given by Proposition 3.5, and let $\left(M^{\prime}, \sigma^{\prime}, g^{\prime}\right)$ be an almost quaternionic hermitian manifold. We say that a Riemannian submersion $\pi: M \rightarrow M^{\prime}$ is a $Q R$ 3-submersion if the following conditions are satisfied:

(i) $\operatorname{Ker} \pi_{*}=\mathcal{V}$;

(ii) for each $p \in M, \sigma_{\pi(p)}^{\prime}$ admits a canonical local basis $\left\{J_{1}^{\prime}, J_{2}^{\prime}, J_{3}^{\prime}\right\}$ such that

$$
\pi_{*} \phi_{\alpha}=J_{\alpha}^{\prime} \pi_{*}, \quad \forall \alpha \in\{1,2,3\} .
$$

REMARK 4.2. We recall that the sections of $\mathcal{V}$, respectively $\mathcal{H}$, are called vertical, respectively horizontal, vector fields. A Riemannian submersion $\pi$ : $M \rightarrow M^{\prime}$ determines two $(1,2)$ tensor fields $T$ and $A$ on $M$ by the formulas

$$
\begin{aligned}
& T(E, F)=T_{E} F=h \nabla_{v E} v F+v \nabla_{v E} h F, \\
& A(E, F)=A_{E} F=v \nabla_{h E} h F+h \nabla_{h E} v F,
\end{aligned}
$$

for any $E, F \in \Gamma(T M)$, where $v$ and $h$ are the vertical and horizontal projections (see $[\mathrm{KO}, \mathrm{ON}]$ ).

We remark that for $U, V \in \Gamma(\mathcal{V}), T_{U} V$ coincides with the second fundamental form of the immersion of the fiber submanifolds, and for $X, Y \in$ $\Gamma(\mathcal{H}), A_{X} Y=\frac{1}{2} v[X, Y]$, reflecting the complete integrability of the horizontal distribution $\mathcal{H}$.

A horizontal vector field $X$ on $M$ is said to be basic if $X$ is $\pi$-related to a vector field $X^{\prime}$ on $M^{\prime}$. It is clear that every vector field $X^{\prime}$ on $M^{\prime}$ has a unique horizontal lift $X$ to $M$, and $X$ is basic.

REMARK 4.3. If $\pi: M \rightarrow M^{\prime}$ is a Riemannian submersion and $X, Y$ are basic vector fields on $M, \pi$-related to $X^{\prime}$ and $Y^{\prime}$ on $M^{\prime}$, then we have the following properties (see [BES, $[\mathrm{FIP}, \mathrm{ON}]$ ):

(i) $h[X, Y]$ is a basic vector field and $\pi_{*} h[X, Y]=\left[X^{\prime}, Y^{\prime}\right] \circ \pi$;

(ii) $h\left(\nabla_{X} Y\right)$ is a basic vector field $\pi$-related to $\nabla_{X^{\prime}}^{\prime} Y^{\prime}$, where $\nabla$ and $\nabla^{\prime}$ are the Levi-Civita connections on $M$ and $M^{\prime}$;

(iii) $[E, U] \in \Gamma(\mathcal{V})$ for all $U \in \Gamma(\mathcal{V})$ and $E \in \Gamma(T M)$. 
Proposition 4.4. Let $M$ be a mixed geodesic QR-hypersurface of a quaternionic Kähler manifold $(\bar{M}, \bar{\sigma}, \bar{g})$ and let $\left(M^{\prime}, \sigma^{\prime}, g^{\prime}\right)$ be an almost quaternionic hermitian manifold. If $\pi: M \rightarrow M^{\prime}$ is a QR 3-submersion, then the distributions $\mathcal{V}$ and $\mathcal{H}$ are invariant by $\phi_{\alpha}$ for all $\alpha \in\{1,2,3\}$.

Proof. Let $V \in \Gamma(\mathcal{V})$. Then

$$
\pi_{*} \phi_{\alpha} V=J_{\alpha}^{\prime} \pi_{*} V=0
$$

and so $\phi_{\alpha}(\mathcal{V}) \subset \mathcal{V}$.

On the other hand, for any $X \in \Gamma(\mathcal{H})$ and $V \in \Gamma(\mathcal{V})$, we derive from (3.8) that

$$
g\left(\phi_{\alpha} X, V\right)=-g\left(X, \phi_{\alpha} V\right)=0,
$$

and thus $\phi_{\alpha}(\mathcal{H}) \subset \mathcal{H}$.

TheOREM 4.5. Let $\pi: M \rightarrow M^{\prime}$ be a QR 3-submersion such that the canonical almost contact 3-structure $\left(\phi_{\alpha}, \xi_{\alpha}, \eta_{\alpha}\right)_{\alpha \in\{1,2,3\}}$ on $M$ is a 3cosymplectic structure. Then $M^{\prime}$ is locally hyper-Kähler.

Proof. For any local basic vector fields $X, Y$ on $M, \pi$-related to $X^{\prime}$ and $Y^{\prime}$ on $M^{\prime}$, from $(3.9)$ we have

$$
\nabla_{X} \phi_{\alpha} Y-\phi_{\alpha} \nabla_{X} Y=0, \quad \forall \alpha \in\{1,2,3\} .
$$

and from 4.3 we deduce

$$
\pi_{*}\left(\nabla_{X} \phi_{\alpha} Y\right)-\pi_{*} \phi_{\alpha} \nabla_{X} Y=0, \quad \forall \alpha \in\{1,2,3\} .
$$

Thus, since $Y$ is a basic vector field $\pi$-related to $Y^{\prime}$, also $\phi_{\alpha} Y$ is basic and $\pi$-related to $J_{\alpha}^{\prime} Y^{\prime}$, and taking account of Definition 4.1 and Remark 4.3, we deduce from 4.4 that

$$
\nabla_{X^{\prime}}^{\prime} J_{\alpha}^{\prime} Y^{\prime}-J_{\alpha}^{\prime} \nabla_{X^{\prime}}^{\prime} Y^{\prime}=0, \quad \forall \alpha \in\{1,2,3\},
$$

thus $\left(\nabla_{X^{\prime}}^{\prime} J_{\alpha}^{\prime}\right) Y^{\prime}=0$, and so $M^{\prime}$ is locally hyper-Kähler.

COROLLARY 4.6. Let $M$ be a totally geodesic QR-hypersurface of a quaternionic Kähler manifold $(\bar{M}, \bar{\sigma}, \bar{g})$, and $\left(M^{\prime}, \sigma^{\prime}, g^{\prime}\right)$ be an almost quaternionic hermitian manifold. If $\pi: M \rightarrow M^{\prime}$ is a $Q R$ 3-submersion such that $\xi_{1}, \xi_{2}$ and $\xi_{3}$ are parallel in $M$, then $M^{\prime}$ is locally hyper-Kähler.

Proof. In this case $\left(\phi_{\alpha}, \xi_{\alpha}, \eta_{\alpha}\right)_{\alpha \in\{1,2,3\}}$ is a 3-cosymplectic structure on $M$ (see [GS]) and the proof is obvious from Theorem 4.5 .

TheOREM 4.7. Let $M$ be a mixed geodesic QR-hypersurface of a quaternionic Kähler manifold $(\bar{M}, \bar{\sigma}, \bar{g}),\left(M^{\prime}, \sigma^{\prime}, g^{\prime}\right)$ be an almost quaternionic hermitian manifold and $\pi: M \rightarrow M^{\prime}$ be a QR 3-submersion. If the natural almost contact metric 3 -structure $\left(\phi_{\alpha}, \xi_{\alpha}, \eta_{\alpha}\right)_{\alpha \in\{1,2,3\}}$ on $M$ is 3-cosymplectic, then the fiber submanifolds are totally geodesic immersed and the horizontal distribution is integrable. 
Proof. Since $M$ is 3-cosymplectic we have

$$
\nabla_{U} \phi_{\alpha} V=\phi_{\alpha} \nabla_{U} V, \quad \forall \alpha \in\{1,2,3\},
$$

for all $U, V \in \Gamma(\mathcal{V})$. Taking the horizontal components, we obtain

$$
T_{U} \phi_{\alpha} V=\phi_{\alpha} T_{U} V, \quad \forall \alpha \in\{1,2,3\},
$$

which immediately implies

$$
T_{U} V=-T_{\phi_{\alpha} U} \phi_{\alpha} V, \quad \forall \alpha \in\{1,2,3\} .
$$

From (4.7), taking account of (3.6), we obtain $T=0$. Similarly we obtain $A=0$ and the proof is now complete, via Remark 4.2 .

Let $M$ be an orientable submanifold of a Riemannian manifold $(\bar{M}, \bar{g})$. We say that $M$ is a totally umbilical submanifold of $\bar{M}$ if the second fundamental form $h$ of $M$ satisfies

$$
h(E, F)=g(E, F) H, \quad \forall E, F \in \Gamma(T M),
$$

where $H$ is the mean curvature vector field on $M$. Moreover, if $H$ is non-zero and parallel in the normal bundle $T M^{\perp}$, then $M$ is called an extrinsic sphere.

By using the Gauss equation, 2.4 and the Gray-O'Neill equation (see $[\mathrm{BES}, \mathrm{FIP}, \mathrm{MNG}, \mathrm{ON}]$ ), we can easily prove the next result.

THEOREM 4.8. Let $M$ be a $Q R$ extrinsic hypersphere of a flat quaternionic Kähler manifold $(\bar{M}, \bar{\sigma}, \bar{g})$ and let $\left(M^{\prime}, \sigma^{\prime}, g^{\prime}\right)$ be another quaternionic Kähler manifold. If $\pi: M \rightarrow M^{\prime}$ is a QR 3-submersion, then $M^{\prime}$ is a quaternionic space form.

EXAmple 4.9. Let $S^{4 m+3}$ be the standard hypersphere in $\mathbb{R}^{4 m+4}$. Then the canonical mapping $\pi: S^{4 m+3} \rightarrow P^{m}(\mathbb{H})$ is a QR 3-submersion.

\section{Quaternionic submersions}

Definition 5.1 ([IMV1]). Let $(M, \sigma, g)$ and $\left(N, \sigma^{\prime}, g^{\prime}\right)$ be two almost quaternionic hermitian manifolds. A map $f: M \rightarrow N$ is said to be $\left(\sigma, \sigma^{\prime}\right)$ holomorphic at a point $x \in M$ if for any $J \in \sigma_{x}$ there exists $J^{\prime} \in \sigma_{f(x)}^{\prime}$ such that $f_{*} \circ J=J^{\prime} \circ f_{*}$. Moreover, we say that $f$ is $\left(\sigma, \sigma^{\prime}\right)$-holomorphic if it is $\left(\sigma, \sigma^{\prime}\right)$-holomorphic at each $x \in M$.

Definition 5.2 ([IMV2]). Let $(M, \sigma, g)$ and $\left(N, \sigma^{\prime}, g^{\prime}\right)$ be two almost quaternionic hermitian manifolds. A Riemannian submersion $\pi: M \rightarrow N$ which is a $\left(\sigma, \sigma^{\prime}\right)$-holomorphic map is called a quaternionic submersion.

Theorem 5.3. Let $\pi:(M, \sigma, g) \rightarrow\left(N, \sigma^{\prime}, g^{\prime}\right)$ be a quaternionic submersion such that $(M, \sigma, g)$ is a quaternionic Kähler manifold. Then $\left(N, \sigma^{\prime}, g^{\prime}\right)$ is a quaternionic Kähler manifold.

Proof. Let $X_{*}, Y_{*} \in \Gamma(T N)$ be such that $\pi_{*} X=X_{*}, \pi_{*} Y=Y_{*}$, where $X, Y \in \Gamma(T M)$. Then 


$$
\begin{aligned}
\left(\nabla_{X_{*}}^{\prime} J_{\alpha}^{\prime}\right) Y_{*} & =\nabla_{X_{*}}^{\prime}\left(J_{\alpha}^{\prime} Y_{*}\right)-J_{\alpha}^{\prime}\left(\nabla_{X_{*}}^{\prime} Y_{*}\right) \\
& =\nabla_{\pi_{*} X}^{\prime}\left(J_{\alpha}^{\prime} \pi_{*} Y\right)-J_{\alpha}^{\prime}\left(\nabla_{\pi_{*} X}^{\prime} \pi_{*} Y\right) \\
& =\nabla_{\pi_{*} X}^{\prime}\left(\pi_{*}\left(J_{\alpha} Y\right)\right)-J_{\alpha}^{\prime} \pi_{*}\left(h \nabla_{X} Y\right) \\
& =\pi_{*}\left(h \nabla_{X}\left(J_{\alpha} Y\right)\right)-\pi_{*}\left(J_{\alpha}\left(h \nabla_{X} Y\right)\right)=\pi_{*}\left(\left(\nabla_{X} J_{\alpha}\right) Y\right) .
\end{aligned}
$$

Since $(M, \sigma, g)$ is a quaternionic Kähler manifold we have $(2.3)$ and we can define 1-forms $\omega_{1}^{\prime}, \omega_{2}^{\prime}, \omega_{3}^{\prime}$ on $N$ by

$$
\omega_{\alpha}^{\prime}\left(X_{*}\right) \circ \pi=\omega_{\alpha}(X), \quad \forall \alpha \in\{1,2,3\},
$$

for any local vector field $X_{*}$ on $N$ and $X$ a real basic vector field on $M$ such that $\pi_{*} X=X_{*}$.

From (2.3), (5.1) and (5.2) we deduce that for all $\alpha \in\{1,2,3\}$,

$$
\left(\nabla_{X_{*}}^{\prime} J_{\alpha}^{\prime}\right) Y_{*}=\omega_{\alpha+2}^{\prime}\left(X_{*}\right) J_{\alpha+1}^{\prime} Y_{*}-\omega_{\alpha+1}^{\prime}\left(X_{*}\right) J_{\alpha+2}^{\prime} Y_{*}
$$

for any local vector fields $X_{*}, Y_{*}$ on $M^{\prime}$, where the indices are taken from $\{1,2,3\}$ modulo 3 . Thus we conclude that $\left(N, \sigma^{\prime}, g^{\prime}\right)$ is a quaternionic Kähler manifold.

Corollary 5.4. Let $\pi:(M, \sigma, g) \rightarrow\left(N, \sigma^{\prime}, g^{\prime}\right)$ be a quaternionic submersion such that $(M, \sigma, g)$ is a quaternionic Kähler manifold. Then both $(M, \sigma, g)$ and $\left(N, \sigma^{\prime}, g^{\prime}\right)$ are locally hyper-Kähler manifolds.

Proof. In this case the vertical and horizontal distributions are both integrable (see [IMV2]) and so we can easily conclude that $(M, \sigma, g)$ is a locally hyper-Kähler manifold. The assertion now follows from the above theorem.

COROLLARY 5.5. There are no quaternionic submersions between quaternionic Kähler manifolds which are not locally hyper-Kähler.

Proof. The assertion is obvious from the above corollary.

Acknowledgments. The author expresses his gratitude to the referee for carefully reading the manuscript and giving useful comments. This research was partly supported by CNCSIS-UEFISCSU, project PNII-IDEI code $8 / 2008$, contract no. 525/2009.

\section{References}

[AG] C. Agut, On a class of QR-submanifolds of quaternion space forms, Publ. Math. 54 (1999), 451-456.

[AL] D. V. Alekseevsky, Riemannian spaces with exceptional holonomy groups, Funktsional. Anal. i Prilozhen. 2 (1968), no. 2, 1-10 (in Russian).

[BEJ] C. L. Bejan, On QR-submanifolds of a quaternion Kählerian manifold, Tensor 46 (1987), 411-417.

[BJC] A. Bejancu, QR-submanifolds of quaternion Kaehler manifolds, Chinese J. Math. 14 (1986), 81-94. 
[BF] A. Bejancu and H. R. Farran, On totally umbilical QR-submanifolds of quaternionic Kählerian manifolds, Bull. Austral. Math. Soc. 62 (2000), 95-103.

[BES] A. Besse, Einstein Manifolds, Springer, Berlin, 1987.

[BLR] D. E. Blair, Contact Manifolds in Riemannian Geometry, Lecture Notes in Math. 509, Springer, 1976.

[FIP] M. Falcitelli, S. Ianuş and A. M. Pastore, Riemannian Submersions and Related Topics, World Sci., Singapore, 2004.

[GS] R. Güneş, B. Şahin and S. Keleş, QR-Submanifolds and almost contact 3-structure, Turkish J. Math. 24 (2000), 239-250.

[IMV1] S. Ianuş, R. Mazzocco and G. E. Vîlcu, Harmonic maps between quaternionic Kähler manifolds, J. Nonlinear Math. Phys. 15 (2008), 1-8.

[IMV2] - - - 一, Riemannian submersions from quaternionic manifolds, Acta Appl. Math. 104 (2008), 83-89.

[ISH] S. Ishihara, Quaternion Kählerian manifolds, J. Differential Geom. 9 (1974), 483-500.

[KP] H. S. Kim and J. S. Pak, QR-submanifolds of maximal QR-dimension in quaternionic projective space, J. Korean Math. Soc. 42 (2005), 655-672.

[KPK] J. H. Kwon and J. S. Pak, $Q R$-submanifolds of $(p-1) Q R$-dimension in a quaternionic projective space $Q P^{(n+p) / 4}$, Acta Math. Hungar. 86 (2000), 89-116.

[KO] S. Kobayashi, Submersion of CR-submanifolds, Tohoku Math. J. 39 (1987), 95100.

[KUO] Y. Y. Kuo, On almost contact 3-structure, ibid. 22 (1970), 325-332.

[MNG] V. Mangione, QR-Hypersurfaces of quaternionic Kähler manifolds, Balkan J. Geom. Appl. 8 (2003), 63-70.

[ON] B. O'Neill, Fundamental equations of submersions, Michigan Math. J. 39 (1966), 459-464.

[SHN1] B. Şahin, On QR-submanifolds of a quaternionic space form, Turkish J. Math. 25 (2001), 413-425.

[SHN2] -, QR-lightlike submanifolds of indefinite quaternion Kaehler manifold, Indian J. Pure Appl. Math. 33 (2002), 1685-1706.

[SLM] S. Salamon, Quaternionic Kähler manifolds, Invent. Math. 67 (1982), 143-171.

[WTS] B. Watson, Almost contact metric 3-submersions, Int. J. Math. Math. Sci. 7 (1984), 667-688.

Gabriel Eduard Vîlcu

Department of Mathematics and Computer Science

Petroleum-Gas University of Ploieşti

Bulevardul Bucureşti 39

Ploieşti 100680, Romania

E-mail: gvilcu@mail.upg-ploiesti.ro

and

Research Center in Geometry, Topology and Algebra

Faculty of Mathematics and Computer Science

University of Bucureşti

Str. Academiei 14

Bucureşti 70109, Romania 
